\title{
An Unusual Case of Cecal Mesenteric Dedifferentiated Liposarcoma Involving the Ileocolic Artery Resected by Right Hemicolectomy
}

\author{
DONGWEI ZHANG ${ }^{1,2}$, WILLIAM D. WILLIAMS ${ }^{3}$ and JINPING LAI ${ }^{2}$ \\ ${ }^{1}$ Department of Pathology and Laboratory Medicine, University of \\ Rochester Medical Center, Rochester, NY, U.S.A.; \\ ${ }^{2}$ Department of Pathology, Immunology and Laboratory of Medicine, \\ University of Florida College of Medicine, Gainesville, FL, U.S.A.; \\ ${ }^{3}$ Department of Pathology and Laboratory Medicine, Broward Health North, Deerfield Beach, FL, U.S.A.
}

\begin{abstract}
Background: Colonic mesenteric dedifferentiated liposarcoma is a rare entity. Case Report: A 65-year-old male developed a $6 \mathrm{~cm}$ diameter right-side intra-abdominal mesenteric mass. A biopsy of the mass was performed and the pathology diagnosis was a spindle cell neoplasm most consistent with low-grade sarcoma. The mass was found to involve the ileocolic vessels in the mesentery on the right side of the abdomen, and therefore the patient received right hemicolectomy with mesenteric mass resection. Pathology confirmed a dedifferentiated liposarcoma (DDLPS) arising in a well-differentiated liposarcoma/atypical lipomatous tumor with myxoid changes. The tumor cells were positive for MDM2 amplification by fluorescence in situ hybridization. After 6 months of follow-up, our patient showed no sign of recurrence. Conclusion: We report an unusual cecal mesenteric DDLPS which was successfully resected by right hemicolectomy. The results suggest that DDLPS should be considered in the differential diagnosis of patients presenting with a spindle cell neoplasm in the mesentery.
\end{abstract}

Atypical lipomatous tumor/well differentiated liposarcoma is a lobulated aggressive mesenchymal neoplasm, characterized by mature lymphocytic proliferation showing significant variation in cell size and focal nuclear atypia (1). Dedifferentiated liposarcoma (DDLPS) is a typically nonlipogenic sarcoma that usually arises from an atypical

Correspondence to: Jinping Lai, MD, Ph.D., Department of Pathology, Immunology and Laboratory of Medicine, University of Florida College of Medicine, P.O. Box 100275, Gainesville, FL 32610, U.S.A. Tel: +1 3526279240, e-mail: jinpinglai@ufl.edu

Key Words: Dedifferentiated liposarcoma, cecal mesentery, right hemicolectomy, MDM2. lipomatous tumor/well-differentiated liposarcoma. It typically occurs in older adults, and in up to $10 \%$ of well-differentiated liposarcomas (2). The most common site of DDLPS is the retroperitoneum soft tissue, followed by the extremities, testis and spermatic cord $(3,4)$. DDLPS usually presents as a large painless mass, which is generally incidentally found (5). DDLPSs occurring in the mesentery of small and large intestine is very unusual and have been rarely reported $(6,7)$. Here we report a rare case of DDLPS arising from the cecal mesentery, presenting a $6 \mathrm{~cm}$ intra-abdominal mass.

\section{Case Report}

A 65-year-old male who had past medical history of nonischemic cardiomyopathy, mitral valve replacement, diabetes and hypertension presented for evaluation of abdominal pain. $\mathrm{He}$ also had history of multiple surgeries including right inguinal hernia repair, right total hip replacement, and cholecystectomy. A $6 \mathrm{~cm}$ diameter intra-abdominal mesenteric mass was detected by a computed tomography (CT) scan (Figure 1A). A biopsy was performed and the histologic and immunohistochemical features suggested that it was a lowgrade sarcoma. The tumor appeared to involve the ileocolic vessels. A surgical decision was made and the patient received right hemicolectomy with ileocolic mesenteric mass resection. Grossly, a $6 \times 3 \times 3 \mathrm{~cm}$ well circumscribed solid mass with a thin fibrous pseudocapsule was identified in the mesentery of the cecum, at $1 \mathrm{~cm}$ distal to the ileocecal valve, and $17 \mathrm{~cm}$ from the distal margin. The tumor was homogenously whiteyellow in color, with subtle fibrous septa visible. No necrosis was grossly identified (Figure 1B).

Microscopically, sections of this mass showed a spindle cell neoplasm with moderate nuclear pleomorphism in a fibromyxoid background. Mitotic figures were rarely seen (1/10 high power field). No tumor necrosis or lymphovascular 
invasion was identified (Figure 2A, B). Areas with welldifferentiated liposarcoma/atypical lipomatous tumor were identified. Atypical lipoblasts were also identified at the inked margin as well as at the subserosal surface of the cecum. The sections of the tumor also showed some immature fat with lipoblasts present in the septa. Mast cells were frequently seen in the tumor. Immunohistochemically, the tumor cells were strongly positive for MDM2, vimentin and p16, weakly positive for smooth muscle actin and caldesmon, and focally positive for CD34. The tumor cells were negative for cytokeratins AE1/AE3, ALK1, desmin, CD117, SOX10, and S100. CD117 and tryptase highlighted many mast cells in the tumor. Beta-catenin showed membranous staining. Ki-67 showed a low proliferation index $(<5 \%)$. Fluorescence in situ hybridization was performed and the tumor cells were positive for MDM2 amplification (Figure 2C and D).

Based on the histologic features, immunoprofile and MDM2 fluorescence in situ hybridization results the final pathologic diagnosis was low-grade (Histologic Grade (French Federation of Cancer Centers Sarcoma Group FNCLCC): Grade 1) dedifferentiated liposarcoma arising in a well-differentiated liposarcoma/atypical lipomatous tumor with myxoid changes. The patient recovered well and was discharged quickly. At 6month follow-up, the patient was doing well and there was no indication of tumor recurrence (all imaging studies were negative and there was no bowel obstruction).

\section{Discussion}

DDLPS is most commonly seen in the retroperitoneum, extremities and trunk, and scrotum/spermatic cord, but rarely seen in the mesentery (8). The differential diagnoses of this case include spindle cell neoplasms involving the mesentery such as intra-abdominal fibromatosis (desmoid tumor), reactive nodular fibrous pseudotumor, inflammatory myofibroblastic tumor, and sclerosing mesenteritis. Fibromatosis usually shows an infiltrative locally aggressive growth pattern. The tumor cells are relatively bland and more collagenous without significant cytologic atypia or mitosis. Fibromatosis is characterized by mutations in the beta-catenin gene or the adenomatous polyposis coli gene, most are sporadic but some are associated with several syndromes such as Gardner's syndrome. Most fibromatoses show diffuse nuclear beta-catenin which is helpful to make the diagnosis (9). Reactive nodular fibrous pseudotumor is a benign reactive fibroinflammatory process involving the gastrointestinal tract and mesentery, and is more common in men. It is a cellular lesion composed of stellate or spindled fibroblasts arranged haphazardly or in intersecting fascicles with collagen-rich stroma. The lesional cells are positive for cytokeratin, CD117 and muscle specific actin (10). Inflammatory myofibroblastic tumor is usually seen in children and young adults. The tumor cells are spindle with abundant amphophilic cytoplasm. There is prominent lymphoplasmacytic infiltration and fibrosis in the tumor. Half of the inflammatory myofibroblastic tumors are positive for ALK1 (11). Sclerosing mesenteritis is a spectrum of idiopathic primary inflammatory and fibrotic processes that affect the mesentery. The lesion shows fibrosis with massive accumulation of thick collagen, fat necrosis, chronic inflammation especially around the vessels and variable focal calcification. The diagnosis of sclerosing mesenteritis is based on hematoxylin and eosin staining and excluding other disorders. There is no specific immunohistochemical stain for it (12).

MDM2 amplification has been estimated to occur in the majority of well-differentiated liposarcoma/atypical lipomatous tumors and dedifferentiated liposarcomas ( 98\%) (13). In this case, MDM2 gene locus amplification was detected by MDM2 interphase fluorescence in situ hybridization. Immunohistochemistry showed strong and diffuse MDM2 positive immunoreactivity in the tumor cells. The above ancillary studies confirmed the diagnosis of DDLPS.

Regarding the prognosis of DDLPS, it has been reported that DDLPS occurring in the common sites (retroperitoneum and extremities) is considered a high-grade sarcoma but shows a better prognosis than other pleomorphic sarcomas. It has a local recurrence rate of $40-50 \%$ and a metastatic rate of $15-17 \%(8,14)$. Complete resection of the tumor remains the principal treatment for DDLPS, regardless of tumor size. Our patient recovered uneventfully and was discharged on postoperative day 9. No evidence of recurrence or metastasis was noted on a follow-up CT scan at the 6-month follow-up examination. Due to the limited number of case reports, the prognosis of mesenteric DDLPS is still unknown. Given the aggressive behavior of DDLPS in the common sites, this patient is closely followed-up by CT scan every 3 months.

In conclusion, DDLPS is most commonly seen in the retroperitoneum and extremities, but rarely seen in the mesentery. We reported an unusual cecal mesenteric DDLPS which was successfully resected by right hemicolectomy. The results suggest that DDLPS should be considered in the differential diagnosis of patients presenting with a spindle cell neoplasm in the mesentery.

\section{References}

1 Dei Tos AP: Liposarcoma: new entities and evolving concepts. Ann Diagn Pathol 4(4): 252-266, 2000.

2 Fletcher CDM, Bridge JA, Hogendoorn PCW and Mertens F: WHO Classification of Tumours of Soft Tissue and Bone. Lyon: IARC Press, 37-38, 2013.

3 Fletcher CDM: Soft tissue tumors. In: Diagnostic histopathology of tumors, third edition. Churchill Livingstone Elsevier, Edinburgh, pp. 1527-1592, 2007.

4 Lewis JJ, Leung D, Woodruff JM and Brennan MF: Retroperitoneal soft-tissue sarcoma: analysis of 500 patients treated and followed at a single institution. Ann Surg 228: 355$365,1998$. 

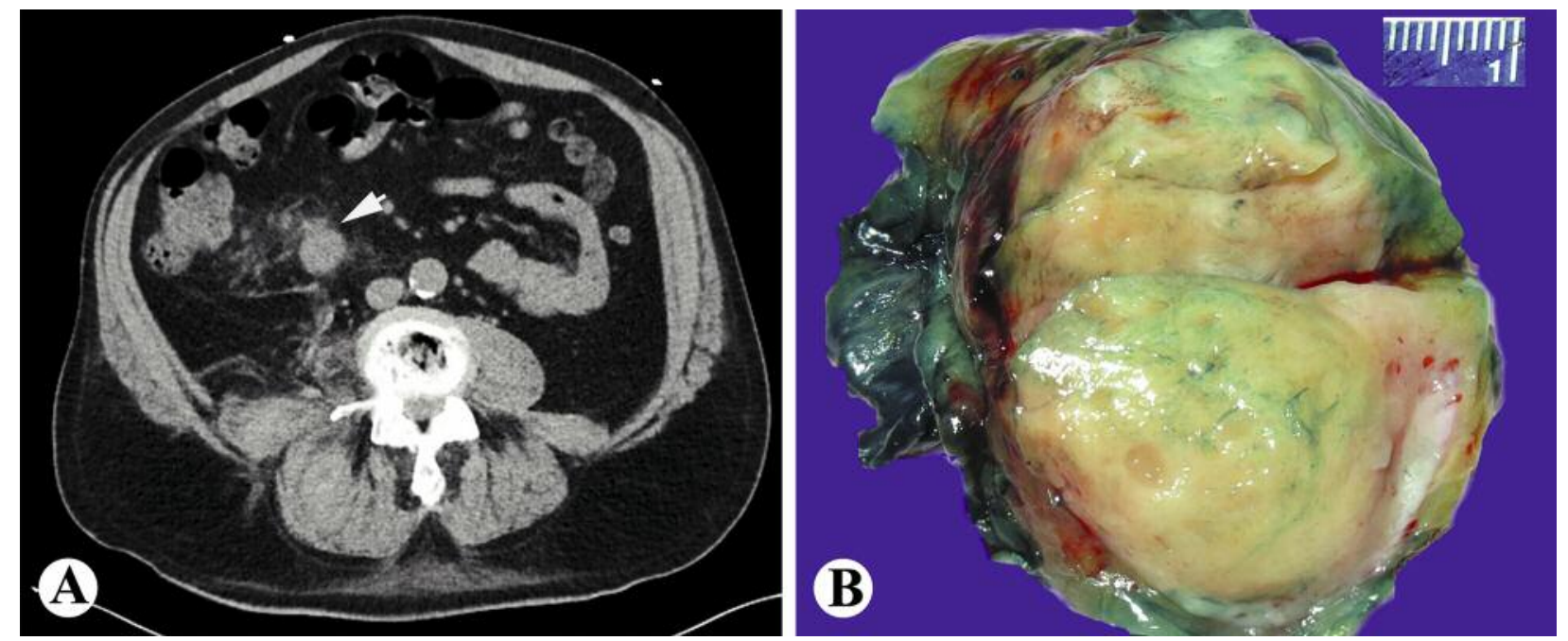

Figure 1. Radiologic finding and gross image of cecal mesenteric DDLPS. A: CT scan showing a $6 \mathrm{~cm}$ intra-abdominal mesenteric mass (arrow). B: Gross picture of the mass in the mesentery of the cecum showing a well circumscribed nonencapsulated solid fatty mass.

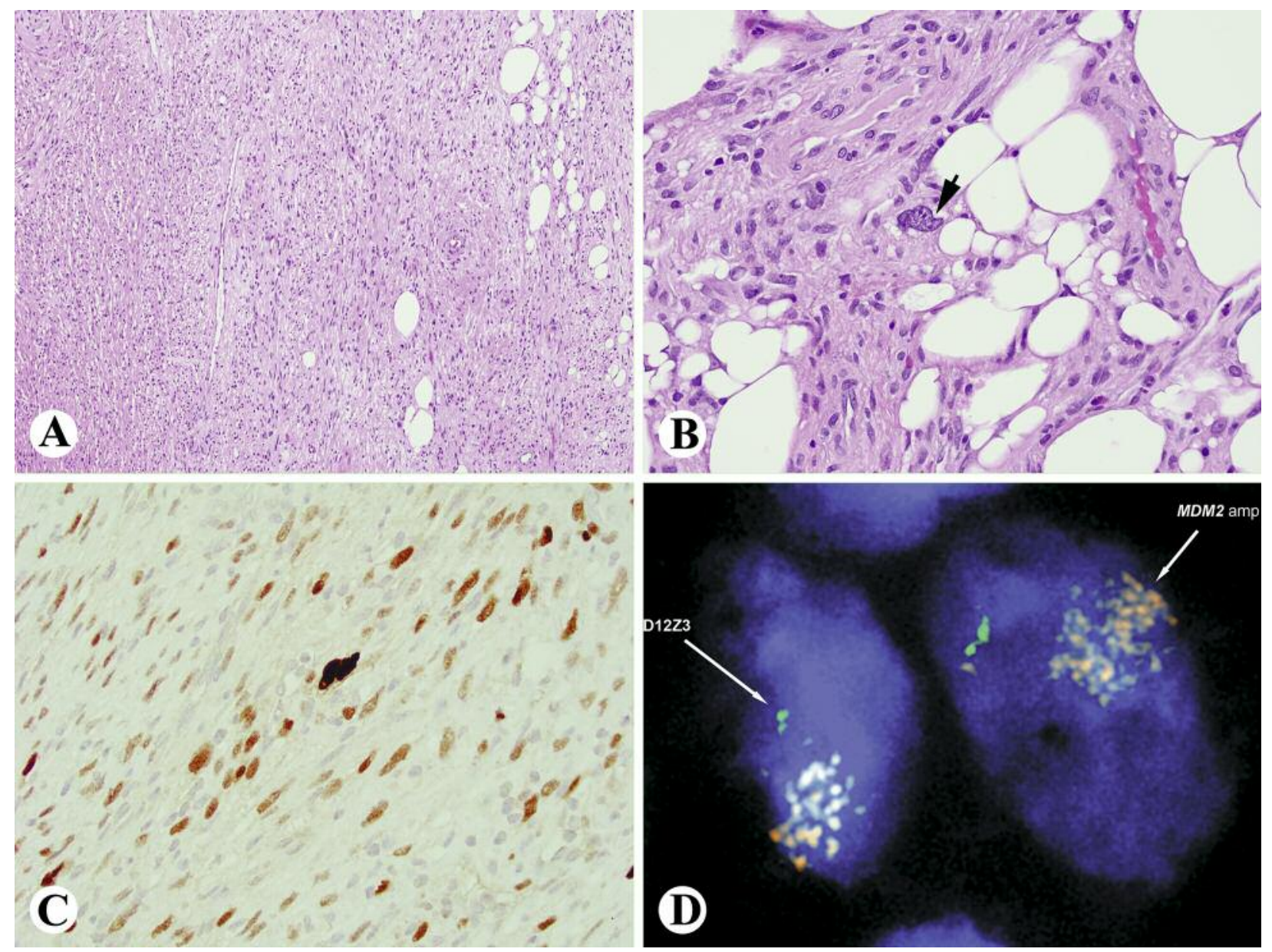

Figure 2. Histological features and ancillary studies of cecal mesenteric DDLPS. A: Low-power view of the tumor showing spindle cells admixed with adipocytes in a fibromyxoid background. HE staining $\times 20$. B: High-power magnification showing nuclear pleomorphism and atypical lipoblast (arrow). HE staining $\times 200 . C, D$ : The diagnosis of DDLPS is confirmed by MDM2 positive immunoreactivity detected by immunohistochemistry (C) and MDM2 amplification deleted by fluorescence in situ hybridization (D). 
5 Weiss SW and Goldblum JR: Enzinger and Weiss's soft tissue tumors, fifth edition. Mosby: St Louis, 2001.

6 Cha EJ: Dedifferentiated liposarcoma of the small bowel mesentery presenting as a submucosal mass. World J Gastrointest Oncol 3(7): 116-118, 2011.

7 Patel J, Deb R, Speake W and Macculloch TA: Primary small bowel liposarcoma (atypical lipomatous tumour) with myogenic differentiation. Sarcoma pii: 807981. doi: 10.1155/2010/807981, 2010.

8 Henricks WH, Chu YC, Goldblum JR and Weiss SW: Dedifferentiated liposarcoma: a clinicopathological analysis of 155 cases with a proposal for an expanded definition of dedifferentiation. Am J Surg Pathol 21(3): 271-281, 1997.

9 Pai SA and Zaveri SS: Intra-abdominal fibromatosis of the jejunum and mesentery. J Clin Pathol 57(10): 1119, 2004.

10 Yantiss RK, Nielsen GP, Lauwers GY and Rosenberg AE: Reactive nodular fibrous pseudotumor of the gastrointestinal tract and mesentery: a clinicopathologic study of five cases. Am J Surg Pathol 27(4): 532-540, 2003.

11 Yamamoto H, Yamaguchi H, Aishima S, Oda Y, Kohashi K, Oshiro $\mathrm{Y}$ and Tsuneyoshi M: Inflammatory myofibroblastic tumor versus IgG4-related sclerosing disease and inflammatory pseudotumor: a comparative clinicopathologic study. Am J Surg Pathol 33(9): 1330-1340, 2009.
12 Emory TS, Monihan JM, Carr NJ and Sobin LH: Sclerosing mesenteritis, mesenteric panniculitis and mesenteric lipodystrophy: a single entity? Am J Surg Pathol 21(4): 392-398, 1997.

13 Weaver J, Downs-Kelly E, Goldblum JR, Turner S, Kulkarni S, Tubbs RR, Rubin BP and Skacel M: Fluorescence in situ hybridization for MDM2 gene amplification as a diagnostic tool in lipomatous neoplasms. Mod Pathol 21(8): 943-949, 2008.

14 McCormick D, Mentzel T, Beham A and Fletcher CD: Dedifferentiated liposarcoma. Clinicopathologic analysis of 32 cases suggesting a better prognostic subgroup among pleomorphic sarcomas. Am J Surg Pathol 18(12): 1213-1223, 1994.

Received November 21, 2018

Revised November 28, 2018

Accepted November 29, 2018 